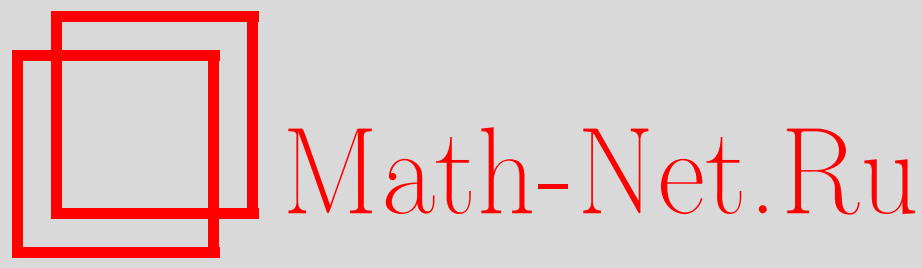

М. Санига, М. Плана, П. Прачна, Проективные кривые над кольцом, включающие в себя два-кубиты, $T M \Phi$, 2008, том 155, номер 3, 463-473

DOI: https://doi.org/10.4213/tmf6223

Использование Общероссийского математического портала Math-Net.Ru подразумевает, что вы прочитали и согласны с пользовательским соглашением http://www.mathnet.ru/rus/agreement

Параметры загрузки:

IP : 18.208 .226 .222

26 апреля 2023 г., 14:41:57

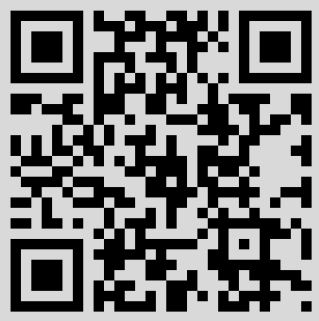




\title{
ФИЗИКА
}

Том 155, № 3

июнь, 2008

\section{8 г.}

\author{
М. Санига* ${ }^{*}$ М. Плана ${ }^{\dagger}$, П. Прачна ${ }^{\ddagger}$
}

\section{ПРОЕКТИВНЫЕ КРИВЫЕ НАД КОЛЬЦОМ, ВКЛЮЧАЮЩИЕ В СЕБЯ ДВА-КУБИТЫ}

Найдено, что проективная прямая над (некоммутативным) кольцом матриц размера $2 \times 2$ с коэффициентами в группе $G F(2)$ полностью включает в себя алгебру 15 операторов (обобщенных матриц Паули), задающую систему два-кубитов. Соответствующая подконфигурация состоит из 15 точек, каждая из которых одновременно оказывается или удаленной, или соседней по отношению к двум (произвольно заданным) удаленным точкам проективной прямой. Операторы можно отождествить с точками таким однозначным образом, чтобы их коммутационные соотношения в точности воспроизводились бы соответствующей геометрией точек; при этом геометрические отношения соседства и удаленности отвечают соответственно операторным отношениям коммутативности и некоммутативности. Эту примечательную конфигурацию можно рассматривать двумя принципиально разными способами, основанными соответственно на базисных $9+6$ и $10+5$ факторизациях алгебры наблюдаемых. Во-первых, эта конфигурация представляет собой объединение взаимно несвязных проективной прямой над $G F(2) \times G F(2)$ ("мерминовская" составляющая) и двух линий над $G F(4)$, проходящих через две выбранные точки, которые мы опустим. Во-вторых, она оказывается обобщенным четырехугольником порядка два, овоиды и/или растяжки которого отвечают соответственно (максимальным) наборам пяти взаимно некоммутирующих операторов и/или группам из пяти максимальных подмножеств коммутирующих операторов, каждое из которых содержит три оператора. Это наблюдение открывает неожиданные возможности для алгебро-геометрического моделирования конечномерных квантовых систем и совершенно новые перспективы для их многообразных применений.

Ключевые слова: проективные прямые над кольцом, обобщенный четырехугольник порядка два, два-кубит.

В последнее время сформировалось понимание того, что проективные прямые, заданные над конечными ассоциативными кольцами с единицей [1]-[7], представляют

*Astronomical Institute, Slovak Academy of Sciences, Tatranská Lomnica, Slovak Republic. E-mail: msaniga@astro.sk

${ }^{\dagger}$ Institut FEMTO-ST, CNRS, Département LPMO, Besançon, France.

E-mail: michel.planat@femto-st.fr

${ }^{\ddagger}$ J. Heyrovský Institute of Physical Chemistry, Academy of Sciences of the Czech Republic, Prague, Czech Republic. E-mail: petr.pracna@jh-inst.cas.cz 
собой новый аппарат, позволяющий глубже проникнуть в алгебро-геометрическую структуру, скрытую в основании конечномерной квантовой системы [8]-[10]. В случае два-кубитов, т.е. для набора из 15 операторов - обобщенных спиновых матриц Паули размера $4 \times 4$, особенное значение приобретают прямые, заданные над прямым произведением простейших полей Галуа $G F(2) \times G F(2) \times \cdots \times G F(2)$. В этом случае прямая, заданная над $G F(2) \times G F(2)$, играет определяющую роль в качественном понимании базисной структуры так называемых квадратов Мермина [9], [10], т.е. массивов размерности $3 \times 3$ в некотором примечательном расщеплении алгебры операторов на факторизации $9+6$, в то время как прямая над $G F(2) \times G F(2) \times G F(2)$ отражает некоторые основные свойства специальной факторизации набора типа $8+7$ ("куб и керн") [10]. Отталкиваясь от этих частных результатов, мы начали поиск такой прямой над кольцом, которая бы содержала полную структуру алгебры всех 15 операторов (матриц). Исследовав достаточно большое число прямых, заданных над коммутативными кольцами [6], [7], мы постепенно пришли к пониманию того, что перспективный кандидат должен, по всей видимости, относиться к некоммутативным объектам, и это, в самом деле, оказалось так. Таким объектом, как будет достаточно подробно продемонстрировано, является проективная прямая, заданная над полным кольцом $(2 \times 2)$-матриц с элементами, лежащими в поле $G F(2),-$ единственным простым некоммутативным кольцом порядка 16 , имеющим шесть единиц (обратимых элементов) и десять делителей нуля [11]. Предпочитая концептуальную часть формальной, мы попытаемся свести к минимуму техническую часть изложения, отсылая интересующегося читателя к соответствующей литературе.

Напомним сначала понятие проективной прямой над кольцом [1]-[7]. Пусть задано ассоциативное кольцо $R$ с единицей [12]-[14]; рассмотрим группу $G L(2, R)$, которая представляет собой общую линейную группу обратимых $(2 \times 2)$-матриц с элементами из $R$. Пара $(a, b) \in R^{2}$ называется допустимой над кольцом $R$, если существуют такие $c, d \in R$, что

$$
\left(\begin{array}{ll}
a & b \\
c & d
\end{array}\right) \in G L(2, R)
$$

Проективной прямой над $R$, обычно обозначаемой $P_{1}(R)$, называется множество классов эквивалентности упорядоченных пар $(\varrho a, \varrho b)$, где $\varrho-$ единица из $R,(a, b)-$ допустимая пара. Две точки $X:=(\varrho a, \varrho b)$ и $Y:=(\varrho c, \varrho d)$ на прямой называются удаленными или соседними, если

$$
\left(\begin{array}{ll}
a & b \\
c & d
\end{array}\right) \in G L(2, R) \quad \text { или } \quad\left(\begin{array}{ll}
a & b \\
c & d
\end{array}\right) \notin G L(2, R)
$$

соответственно. Группа $G L(2, R)$ обладает важным свойством транзитивного действия на наборах из трех попарно удаленных точек: если заданы две тройки взаимно удаленных точек, то существует элемент $G L(2, R)$, переводящий одну тройку в другую. 
Мы будем исследовать только проективную прямую, заданную над полным кольцом $(2 \times 2)$-матриц с коэффициентами из $G F(2)$, т.е.

$$
R=M_{2}(G F(2)) \equiv\left\{\left(\begin{array}{ll}
\alpha & \beta \\
\gamma & \delta
\end{array}\right) \mid \alpha, \beta, \gamma, \delta \in G F(2)\right\}
$$

Маркируя эти матрицы следующим образом:

$$
\begin{aligned}
& 1 \equiv\left(\begin{array}{cc}
1 & 0 \\
0 & 1
\end{array}\right), \quad 2 \equiv\left(\begin{array}{cc}
0 & 1 \\
1 & 0
\end{array}\right), \quad 3 \equiv\left(\begin{array}{ll}
1 & 1 \\
1 & 1
\end{array}\right), \quad 4 \equiv\left(\begin{array}{ll}
0 & 0 \\
1 & 1
\end{array}\right), \\
& 5 \equiv\left(\begin{array}{ll}
1 & 0 \\
1 & 0
\end{array}\right), \quad 6 \equiv\left(\begin{array}{ll}
0 & 1 \\
0 & 1
\end{array}\right), \quad 7 \equiv\left(\begin{array}{ll}
1 & 1 \\
0 & 0
\end{array}\right), \quad 8 \equiv\left(\begin{array}{ll}
0 & 1 \\
0 & 0
\end{array}\right) \text {, } \\
& 9 \equiv\left(\begin{array}{ll}
1 & 1 \\
0 & 1
\end{array}\right), \quad 10 \equiv\left(\begin{array}{ll}
0 & 0 \\
1 & 0
\end{array}\right), \quad 11 \equiv\left(\begin{array}{ll}
1 & 0 \\
1 & 1
\end{array}\right), \quad 12 \equiv\left(\begin{array}{ll}
0 & 1 \\
1 & 1
\end{array}\right) \text {, } \\
& 13 \equiv\left(\begin{array}{ll}
1 & 1 \\
1 & 0
\end{array}\right), \quad 14 \equiv\left(\begin{array}{ll}
0 & 0 \\
0 & 1
\end{array}\right), \quad 15 \equiv\left(\begin{array}{ll}
1 & 0 \\
0 & 0
\end{array}\right), \quad 0 \equiv\left(\begin{array}{ll}
0 & 0 \\
0 & 0
\end{array}\right) \text {, }
\end{aligned}
$$

можно явно проверить, что сложение и умножение в $M_{2}(G F(2))$ выполняется по правилам, приведенными в табл. 1 (см. [15], с. 433, 531). Проверяя сначала условие совместности (1), а затем, группируя допустимые пары, лево-пропорциональные единице, в классы эквивалентности (каждый содержит шесть элементов), найдем, что прямая ${ }^{1)} P_{1}\left(M_{2}(G F(2))\right)$ содержит в общей сложности 35 точек со следующими представителями в каждом классе эквивалентности (см. работы [6]-[8] относительно деталей данной методологии, а также разнообразных примеров, иллюстрирующих метод проективной прямой над кольцом):
$(1,1), \quad(1,2)$,
$(1,9)$
(1, 11),
$(1,12), \quad(1,13)$,
$(1,0), \quad(1,3)$
$(1,4)$,
$(1,5)$,
$(1,6)$,
$(1,7)$,
$(1,8), \quad(1,10)$,
$(1,14), \quad(1,15)$,
$(0,1), \quad(3,1)$,
$(4,1)$,
$(5,1)$,
$(6,1)$,
$(7,1)$,
$(8,1), \quad(10,1)$
$(14,1)$,
$(3,4), \quad(3,10), \quad(3,14)$,
$(5,4)$,
$(5,10)$,
$(5,14)$,
$(6,4)$
$(6,10), \quad(6,14)$.

Из таблицы умножения легко увидеть, что представители из первой строки в (5) содержат по две единицы (1 сама, очевидно, представляет собой единицу), а представители из второй и третьей строк содержат одну единицу и один делитель нуля, в то время как все пары в последней строке состоят из делителей нуля. Уже на этом этапе можно показать, какая “доля" прямой $P_{1}\left(M_{2}(G F(2))\right)$ задает подходящее алгебро-геометрическое описание два-кубитов.

Рассмотрим теперь две удаленные точки на прямой. Принимая во внимание вышеупомянутую транзитивность $G L(2, R)$ относительно выбора тройки взаимно удаленных точек, можно, не ограничивая общности, выбрать точки $U:=(1,0)$ и $V:=(0,1)$. Затем выберем все такие точки прямой, которые или одновременно удалены, или одновременно являются соседними точкам $U$ и $V$. При выполнении

\footnotetext{
1)Было обнаружено, что эта прямая определенно относится к некоммутативным прямым над кольцом, поскольку она коренным образом отличается от двух своих коммутативных аналогов [11].
}

4 Теоретическая и математическая физика, т. 155, № 3, 2008 г. 
ТАБлицА 1. Сложение (верхние значения) и умножение (нижние значения) в $M_{2}(G F(2))$

\begin{tabular}{|c|c|c|c|c|c|c|c|c|c|c|c|c|c|c|c|c|}
\hline+ & 0 & 1 & 2 & 3 & 4 & 5 & 6 & 7 & 8 & 9 & 10 & 11 & 12 & 13 & 14 & 15 \\
\hline 0 & 0 & 1 & 2 & 3 & 4 & 5 & 6 & 7 & 8 & 9 & 10 & 11 & 12 & 13 & 14 & 15 \\
\hline 1 & 1 & 0 & 3 & 2 & 5 & 4 & 7 & 6 & 9 & 8 & 11 & 10 & 13 & 12 & 15 & 14 \\
\hline 2 & 2 & 3 & 0 & 1 & 6 & 7 & 4 & 5 & 10 & 11 & 8 & 9 & 14 & 15 & 12 & 13 \\
\hline 3 & 3 & 2 & 1 & 0 & 7 & 6 & 5 & 4 & 11 & 10 & 9 & 8 & 15 & 14 & 13 & 12 \\
\hline 4 & 4 & 5 & 6 & 7 & 0 & 1 & 2 & 3 & 12 & 13 & 14 & 15 & 8 & 9 & 10 & 11 \\
\hline 5 & 5 & 4 & 7 & 6 & 1 & 0 & 3 & 2 & 13 & 12 & 15 & 14 & 9 & 8 & 11 & 10 \\
\hline 6 & 6 & 7 & 4 & 5 & 2 & 3 & 0 & 1 & 14 & 15 & 12 & 13 & 10 & 11 & 8 & 9 \\
\hline 7 & 7 & 6 & 5 & 4 & 3 & 2 & 1 & 0 & 15 & 14 & 13 & 12 & 11 & 10 & 9 & 8 \\
\hline 8 & 8 & 9 & 10 & 11 & 12 & 13 & 14 & 15 & 0 & 1 & 2 & 3 & 4 & 5 & 6 & 7 \\
\hline 9 & 9 & 8 & 11 & 10 & 13 & 12 & 15 & 14 & 1 & 0 & 3 & 2 & 5 & 4 & 7 & 6 \\
\hline 10 & 10 & 11 & 8 & 9 & 14 & 15 & 12 & 13 & 2 & 3 & 0 & 1 & 6 & 7 & 4 & 5 \\
\hline 11 & 11 & 10 & 9 & 8 & 15 & 14 & 13 & 12 & 3 & 2 & 1 & 0 & 7 & 6 & 5 & 4 \\
\hline 12 & 12 & 13 & 14 & 15 & 8 & 9 & 10 & 11 & 4 & 5 & 6 & 7 & 0 & 1 & 2 & 3 \\
\hline 13 & 13 & 12 & 15 & 14 & 9 & 8 & 11 & 10 & 5 & 4 & 7 & 6 & 1 & 0 & 3 & 2 \\
\hline 14 & 14 & 15 & 12 & 13 & 10 & 11 & 8 & 9 & 6 & 7 & 4 & 5 & 2 & 3 & 0 & 1 \\
\hline 15 & 15 & 14 & 13 & 12 & 11 & 10 & 9 & 8 & 7 & 6 & 5 & 4 & 3 & 2 & 1 & 0 \\
\hline$x$ & 0 & 1 & 2 & 3 & 4 & 5 & 6 & 7 & 8 & 9 & 10 & 11 & 12 & 13 & 14 & 15 \\
\hline 0 & 0 & 0 & 0 & 0 & 0 & 0 & 0 & 0 & 0 & 0 & 0 & 0 & 0 & 0 & 0 & 0 \\
\hline 1 & 0 & 1 & 2 & 3 & 4 & 5 & 6 & 7 & 8 & 9 & 10 & 11 & 12 & 13 & 14 & 15 \\
\hline 2 & 0 & 2 & 1 & 3 & 7 & 5 & 6 & 4 & 14 & 12 & 15 & 13 & 9 & 11 & 8 & 10 \\
\hline 3 & 0 & 3 & 3 & 0 & 3 & 0 & 0 & 3 & 6 & 5 & 5 & 6 & 5 & 6 & 6 & 5 \\
\hline 4 & 0 & 4 & 4 & 0 & 4 & 0 & 0 & 4 & 14 & 10 & 10 & 14 & 10 & 14 & 14 & 10 \\
\hline 5 & 0 & 5 & 6 & 3 & 0 & 5 & 6 & 3 & 6 & 3 & 0 & 5 & 6 & 3 & 0 & 5 \\
\hline 6 & 0 & 6 & 5 & 3 & 3 & 5 & 6 & 0 & 0 & 6 & 5 & 3 & 3 & 5 & 6 & 0 \\
\hline 7 & 0 & 7 & 7 & 0 & 7 & 0 & 0 & 7 & 8 & 15 & 15 & 8 & 15 & 8 & 8 & 15 \\
\hline 8 & 0 & 8 & 15 & 7 & 7 & 15 & 8 & 0 & 0 & 8 & 15 & 7 & 7 & 15 & 8 & 0 \\
\hline 9 & 0 & 9 & 13 & 4 & 3 & 10 & 14 & 7 & 8 & 1 & 5 & 12 & 11 & 2 & 6 & 15 \\
\hline 10 & 0 & 10 & 14 & 4 & 0 & 10 & 14 & 4 & 14 & 4 & 0 & 10 & 14 & 4 & 0 & 10 \\
\hline 11 & 0 & 11 & 12 & 7 & 4 & 15 & 8 & 3 & 6 & 13 & 10 & 1 & 2 & 9 & 14 & 5 \\
\hline 12 & 0 & 12 & 11 & 7 & 3 & 15 & 8 & 4 & 14 & 2 & 5 & 9 & 13 & 1 & 6 & 10 \\
\hline 13 & 0 & 13 & 9 & 4 & 7 & 10 & 14 & 3 & 6 & 11 & 15 & 2 & 1 & 12 & 8 & 5 \\
\hline 14 & 0 & 14 & 10 & 4 & 4 & 10 & 14 & 0 & 0 & 14 & 10 & 4 & 4 & 10 & 14 & 0 \\
\hline 15 & 0 & 15 & 8 & 7 & 0 & 15 & 8 & 7 & 8 & 7 & 0 & 15 & 8 & 7 & 0 & 15 \\
\hline
\end{tabular}

первого из условий (2) обнаружим, что в первом семействе лежат следующие шесть точек:

$$
\begin{array}{lll}
C_{1}=(1,1), & C_{2}=(1,2), & C_{3}=(1,9), \\
C_{4}=(1,11), & C_{5}=(1,12), & C_{6}=(1,13),
\end{array}
$$

в то время как второе из условий (2) дает, что второе семейство состоит из следующих девяти точек:

$$
\begin{aligned}
C_{7} & =(3,4), & C_{8} & =(3,10), & C_{9} & =(3,14), \\
C_{10} & =(5,4), & C_{11} & =(5,10), & C_{12} & =(5,14), \\
C_{13} & =(6,4), & C_{14} & =(6,10), & C_{15} & =(6,14) .
\end{aligned}
$$


Снова с учетом условий (2) получим, что точки нашего специального подмножества прямой $P_{1}\left(M_{2}(G F(2))\right)$ связаны между собой способом, указанным в табл. 2. Из этой таблицы явно следует, что каждая точка этой конфигурации имеет шесть соседних и восемь удаленных точек, а максимальное число попарно соседних точек равно трем. Осталось только отождествить эти 15 точек с 15 обобщенными матрицами Паули - операторами два-кубитов (см., например, выражение (1) из работы [10]) следующим образом:

$$
\begin{aligned}
& C_{1}=\sigma_{z} \otimes \sigma_{x}, \quad C_{2}=\sigma_{y} \otimes \sigma_{y}, \quad C_{3}=1_{2} \otimes \sigma_{x}, \\
& C_{4}=\sigma_{y} \otimes \sigma_{z}, \quad C_{5}=\sigma_{y} \otimes 1_{2}, \quad C_{6}=\sigma_{x} \otimes \sigma_{x}, \\
& C_{7}=\sigma_{x} \otimes \sigma_{z}, \quad C_{8}=\sigma_{y} \otimes \sigma_{x}, \quad C_{9}=\sigma_{z} \otimes \sigma_{y}, \\
& C_{10}=\sigma_{x} \otimes 1_{2}, \quad C_{11}=\sigma_{x} \otimes \sigma_{y}, \quad C_{12}=1_{2} \otimes \sigma_{y}, \\
& C_{13}=1_{2} \otimes \sigma_{z}, \quad C_{14}=\sigma_{z} \otimes \sigma_{z}, \quad C_{15}=\sigma_{z} \otimes 1_{2} \text {, }
\end{aligned}
$$

где $1_{2}$ - единичная $(2 \times 2)$-матрица, $\sigma_{x}, \sigma_{y}$ и $\sigma_{z}$ - классические матрицы Паули, а символ " $\otimes$ " обозначает тензорное произведение матриц. Теперь нетрудно проверить явно, что в табл. 2 заданы правильные коммутационные соотношения между этими операторами, где символы “+" и “-” теперь имеют соответствующий смысл некоммутативности и коммутативности. Другими словами, одна и та же матрица инцидентности, заданная в табл. 2, имеет отношение к двум выделенным конфигурациям совершенно различного происхождения: к набору точек проективной прямой над некоторым выделенным конечным кольцом, в котором символы “+" и "-" означают алгебро-геометрическое значение отношения удаленности и соседства, и одновременно к набору операторов над четырехмерным гильбертовым пространством, в котором те же самые символы принимают значение соответственно некоммутативности и коммутативности в операторном смысле.

Эту примечательную конфигурацию можно интерпретировать двумя принципиально различными способами, которые связаны соответственно с базисными $9+6$ (рис. 1 а, в) и $10+5$ (рис. 16, г) факторизациями алгебры наблюдаемых. Первая факторизация есть просто несвязное объединение проективной прямой над $G F(2) \times$ $G F(2)$ и двух линий над $G F(4)$, проходящих через две выбранные точки $U$ и $V$, не указанные явно. Как было детально показано в работах [9], [10], прямая над $G F(2) \times G F(2)$ проявляет качественную структуру магических квадратов Мермина, т.е. таблиц размерности $3 \times 3$, содержащих девять наблюдаемых, попарно коммутирующих в каждой строке и столбце и расставленных таким образом, что свойства их произведений противоречат свойствам соответствующих собственных значений. Две прямые над $G F(4)$ отвечают остающейся двудольной составляющей расщепления, в которой три точки (наблюдаемые) на каждой прямой взаимно удалены (некоммутативны), а каждая точка (наблюдаемая) одной прямой оказывается соседней (коммутирующей) с каждой точкой (наблюдаемой) на второй прямой (см. рис. $1 \mathrm{a}$ ).

Вторая интерпретация включает обобщенный четырехугольник, задающийся геометрией ранга два с отношением инцидентности между точками и прямыми, в которой через две точки проходит максимум одна прямая и для любой точки $X$ и прямой 
ТАБлицА 2. Соотношения удаленности и соседства (соответственно “+” и "-") для точек выбранной конфигурации. Точки упорядочены таким образом, что последние девять (т.е. от $C_{7}$ до $C_{15}$ ) образуют проективную прямую над $G F(2) \times G F(2)($ см. [8]-[10]).

\begin{tabular}{|c|ccc|ccc|ccc|ccc|ccc|}
\hline & $C_{1}$ & $C_{2}$ & $C_{3}$ & $C_{4}$ & $C_{5}$ & $C_{6}$ & $C_{7}$ & $C_{8}$ & $C_{9}$ & $C_{10}$ & $C_{11}$ & $C_{12}$ & $C_{13}$ & $C_{14}$ & $C_{15}$ \\
\hline$C_{1}$ & - & - & - & - & + & + & - & + & + & + & - & + & + & + & - \\
$C_{2}$ & - & - & + & + & - & - & - & + & + & + & + & - & + & - & + \\
$C_{3}$ & - & + & - & + & - & - & + & - & + & - & + & + & + & + & - \\
\hline$C_{4}$ & - & + & + & - & - & - & + & + & - & + & - & + & - & + & + \\
$C_{5}$ & + & - & - & - & - & + & + & - & + & + & + & - & - & + & + \\
$C_{6}$ & + & - & - & - & + & - & + & + & - & - & + & + & + & - & + \\
\hline$C_{7}$ & - & - & + & + & + & + & - & - & - & - & + & + & - & + & + \\
$C_{8}$ & + & + & - & + & - & + & - & - & - & + & - & + & + & - & + \\
$C_{9}$ & + & + & + & - & + & - & - & - & - & + & + & - & + & + & - \\
\hline$C_{10}$ & + & + & - & + & + & - & - & + & + & - & - & - & - & + & + \\
$C_{11}$ & - & + & + & - & + & + & + & - & + & - & - & - & + & - & + \\
$C_{12}$ & + & - & + & + & - & + & + & + & - & - & - & - & + & + & - \\
\hline$C_{13}$ & + & + & + & - & - & + & - & + & + & - & + & + & - & - & - \\
$C_{14}$ & + & - & + & + & + & - & + & - & + & + & - & + & - & - & - \\
$C_{15}$ & - & + & - & + & + & + & + & + & - & + & + & - & - & - & - \\
\hline
\end{tabular}

$\mathcal{L}, X \notin \mathcal{L}$, существует ровно одна проходящая через $X$ прямая, которая пересекается с $\mathcal{L}$ (см. [16]-[20]). Обобщенный четырехугольник, ассоциированный с нашими наблюдаемыми, имеет порядок два, т.е. в нем каждая прямая содержит три точки и каждая точка лежит на трех прямых. Такой четырехугольник на самом деле содержит 15 точек (и, ввиду самодуальности, такое же число прямых), каждая из которых связана прямыми с другими шестью точками (рис. 2 слева). Если удалить из этого четырехугольника один из его овоидов, т.е. один из наборов пяти точек, которые имеют ровно одну общую точку с каждой из прямых (рис. 2 в центре), то останется набор из десяти точек, образующих знаменитый петерсеновский граф (рис. 2 справа) (см. [19], [20]). Пять точек овоида отвечают пяти взаимно удаленным точкам на $P_{1}(G F(4))$ и, соответственно, пяти (т.е. максимально возможному числу) взаимно некоммутирующим наблюдаемым два-кубитам. Если в соответствии с соотношением дуальности выкинуть из четырехугольника растяжку, т.е. набор из пяти попарно несвязных линий, которые задают разбиение множества вершин, то мы получим граф, дуальный петерсеновскому (рис. 3); пять линий растяжки представляют собой не что иное, как пять максимальных подмножеств, каждое из которых состоит из трех взаимно коммутирующих операторов. Как будет показано в отдельной публикации, пять линий каждой такой растяжки несут максимальные коммутирующие подмножества операторов, ассоциированные базисы которых взаимно незацеплены [10], [21]. Это означает, что существование и количество элементов растяжки внутри обобщенного четырехугольника равнозначны существованию и количеству элементов максимального множества взаимно незацепленных базисов в соответствующем гильбертовом пространстве. 


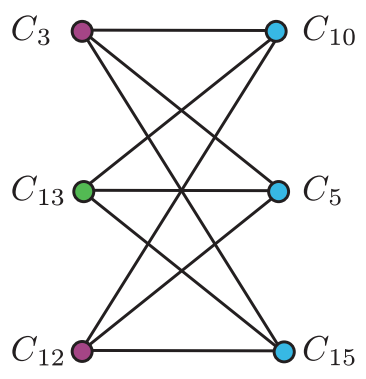

$a$

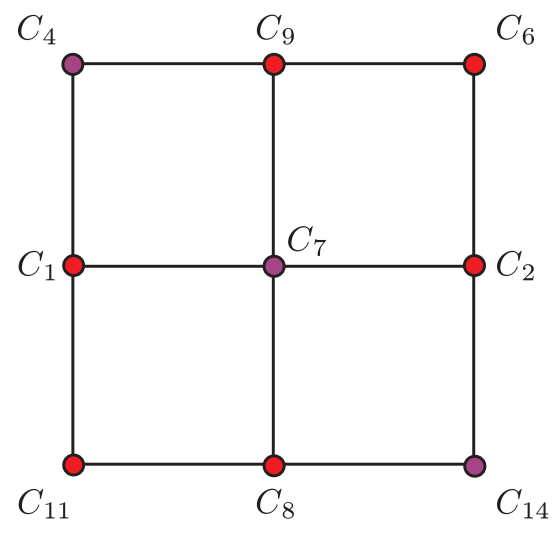

B
$C_{3}$<smiles>[Mg][Mg]</smiles><smiles>[14CH3]O</smiles>

$\sigma$

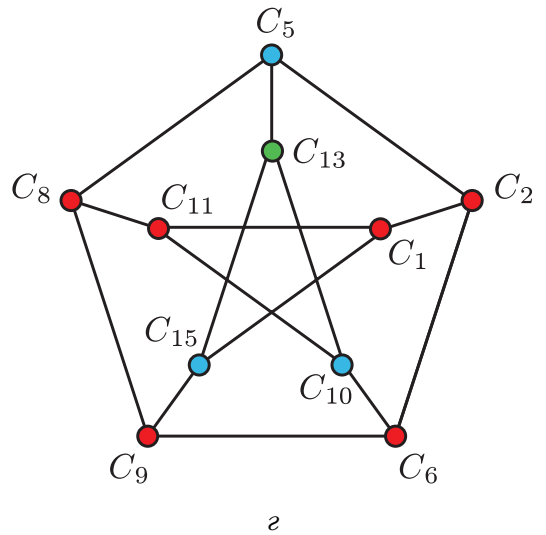

Рис. 1. Две базисных факторизации алгебры 15 наблюдаемых (операторов) системы два-кубитов. Факторизация $9+6$ (а, в) геометрически соответствует расщеплению нашей подсистемы прямой $P_{1}\left(M_{2}(G F(2))\right)$ на проективную прямую над $G F(2) \times G F(2)$ (в) и на пару проективных прямых над $G F(4)$, имеющих две общих точки (а). Расщепление $10+5$ (б, г) отвечает, как также показано другим образом на рис. 2 , расщеплению обобщенного четырехугольника на обобщенный четырехугольник его овоидов (б) и на петерсеновский граф (г). В обоих случаях две точки (наблюдаемые) соединяются линией (сегментом) только, если они оказываются соседними (коммутируют); чтобы не загромождать рисунок мы опускаем ребра, соединяющие точки (наблюдаемые) на двух различных компонентах. Цветовые обозначения (см. электронную версию статьи на сайте SpringerLink) применяются, чтобы показать, как две факторизации связаны друг с другом.

Тем самым такой геометрический подход при его подходящем обобщении на кубиты и кудиты высших порядков предоставляет нам уникальный новый метод для решения проблемы о максимальном числе взаимно незацепленных базисов в конечномерном гильбертовом пространстве, которая до сих пор представляет собой трудную нерешенную задачу в случае, когда эта размерность не равна степени простого 
числа. Теперь осталось выполнить прямое упражнение для того, чтобы увязать точки четырехугольника с операторами (наблюдаемыми) $C_{i}$ (см. уравнение (8)) таким образом, чтобы реконструировать табл. 2 после подстановки знаков "-" или “+”, характеризующих соответственно пары точек четырехугольника, которые лежат или не лежат на одной общей линии.
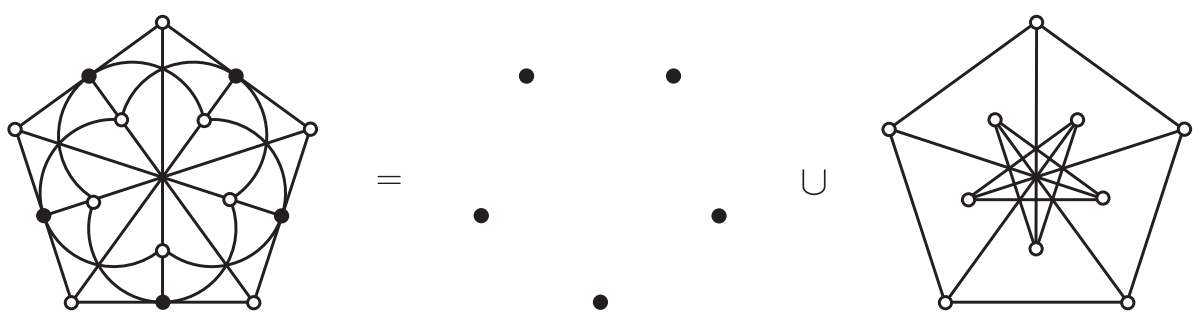

Рис. 2. Обобщенный четырехугольник порядка два (слева) и его факторизация на овоид (в центре) и петерсеновскую часть (справа). Линии четырехугольника показаны прямыми отрезками и сегментами круга; заметим, что не всякое пересечение двух сегментов оказывается точкой четырехугольника.
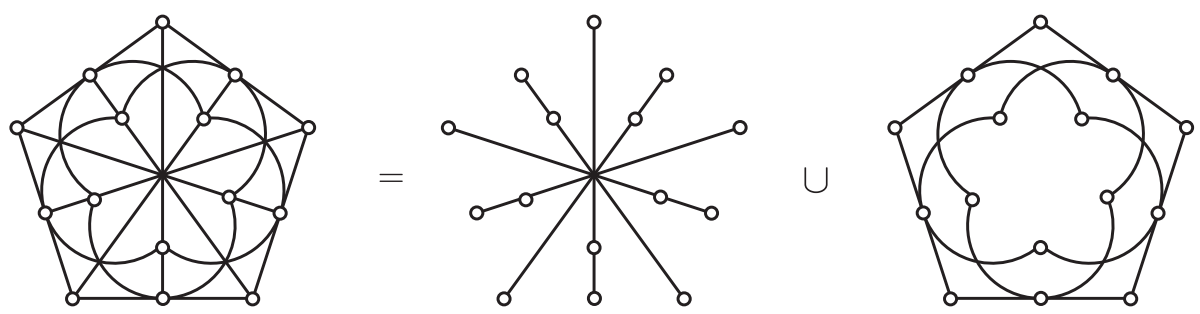

Рис. 3. Дуальный взгляд на обобщенный четырехугольник порядка два (слева) как на несвязное объединение одной из его растяжек (в центре) и графа, дуального петерсеновскому, или 2-растяжки (справа).

Чтобы завершить построение этой интересной алгебро-геометрической картины два-кубитов остается ввести еще один важный геометрический объект. Внимательный читатель, возможно, уже подметил, что нами были использованы два различных типа проективных прямых, определенных над кольцами порядка четыре и характеристики два: одна прямая была задана над полем $G F(4)$, а вторая - над прямым произведением колец $G F(2) \times G F(2)$; первая отвечала овоиду обобщенного четырехугольника, т.е. набору из пяти взаимно некоммутирующих операторов (рис. 1 б), в то время как вторая отвечала решетке из девяти точек на шести линиях $^{2)}$, связанной с квадратом операторов Мермина (рис. 1 в). Однако существует еще одно ассоциативное кольцо с единицей порядка четыре и характеристики два,

2) Также известной как утонъшенный обобщенный четырехугольник порядка $(2,1)($ см., например, работы [18] и [20]). На самом деле обе конфигурации, изображенные на рис. 1 а, в, представляют собой утоньшенные обобщенные четырехугольники, один из которых дуален другому. 
а именно (локальное) кольцо множителей многочленов $G F(2)[x] /\left\langle x^{2}\right\rangle$ (см. [12]-[14]). Это кольцо также является подкольцом $M_{2}(G F(2))$, так что можно ожидать, что соответствующая проективная прямая также играет роль в нашей модели. И это в самом деле так. Как показано, например, в работе [6] (см. табл. 3 указанной работы), проективная прямая $P_{1}\left(G F(2)[x] /\left\langle x^{2}\right\rangle\right)$ содержит шесть точек, каждая из которых оказывается соседней с одной из оставшихся и удаленной от прочих четырех, что тем самым дает три пары соседних точек. В наборе операторов Паули эта конфигурация представляется шестиплетом операторов, коммутирующих с данным оператором; если выбрать, например, $C_{13}$ в качестве последнего, указанные шесть операторов будут представлять собой, как сразу следует из табл. 2, конфигурацию $\left\{C_{4}, C_{5} ; C_{7}, C_{10} ; C_{14}, C_{15}\right\}$, и они в самом деле образуют три пары взаимно коммутирующих членов (эти пары разделены точками с запятыми). В обобщенном четырехугольнике каждая такая конфигурация представляет собой шестиплет точек, соединенных с данной.

Более глубокое понимание и более полное принятие этого наблюдения связано с введением концепции геометрической гиперплоскости. Геометрическая гиперплоскость $H$ в конечной геометрии представляет собой набор точек такой, что каждая линия геометрии или содержит в точности одну точку из $H$, или полностью содержится в $H$ [20], [22]. Нетрудно проверить, что для обобщенного четырехугольника порядка два гиперплоскость $H$ может принадлежать одному из следующих трех классов [22]: 1) $H_{\text {оv }}$ - овоид (имеется шесть таких гиперплоскостей); 2) $H_{\mathrm{cl}}(X)-$ множество точек, соединенных с данной точкой $X$, включая и саму эту точку (имеется 15 таких гиперплоскостей); 3) $H_{\text {gr }}$ - решетка, определенная выше (имеется 10 таких гиперплоскостей). Обнаруживается тем самым превосходное равноправие между тремя возможными типами геометрических гиперплоскостей обобщенного четырехугольника порядка два и тремя возможными типами проективных прямых над кольцами из четырех элементов и характеристики два, погруженными в нашу подконфигурацию прямой $P_{1}\left(M_{2}(G F(2))\right)$, что порождает три возможных типа выделенных подмножеств операторов Паули для два-кубитов (см. табл. 3)

В заключение заметим, что обобщенный четырехугольник порядка два также содержится в прямой $P_{1}\left(M_{2}(G F(2))\right)$ в виде проективной прямой над так называемой жордановой системой симметрических матриц размера $2 \times 2$ над $G F(2)$ [23], или, эквивалентно, в виде обобщенного гиперплоскостного сечения квадрик Клейна в пятимерном проективном пространстве над $G F(2)$ (см. [22]).

Мы показали, что основные свойства системы двух взаимодействующих частиц спина 1/2 единственным образом вкладываются в (под)геометрию некоторой выделенной проективной прямой, которая, как было найдено, эквивалентна обобщенному четырехугольнику порядка два. Поскольку такие системы представляют собой простейшие системы, проявляющие свойства типа квантового зацепления и квантовой нелокальности, они занимают ведущее место в многочисленных приложениях, наиболее популярные из которых квантовая криптография, квантовое кодирование, квантовое клонирование (телепортация) и квантовые вычисления. Наше открытие не только предлагает принципиально новый геометрически обогащенный взгляд на 
ТАБлицА 3. Три типа выделенных подмножеств обобщенных операторов Паули для два-кубитов (TQ), рассматриваемые как геометрические гиперплоскости в обобщенном четырехугольнике порядка два (GQ) и/или как проективные прямые над кольцами порядка четыре и характеристики два, содержащиеся в проективной прямой $P_{1}\left(M_{2}(G F(2))\right)(\mathrm{PL})$.

\begin{tabular}{|c||c|c|c|}
\hline GQ & $H_{\mathrm{ov}}$ & $H_{\mathrm{cl}}(X) \backslash\{X\}$ & $H_{\mathrm{gr}}$ \\
\hline $\mathrm{PL}$ & $P_{1}(G F(4))$ & $P_{1}\left(G F(2)[x] /\left\langle x^{2}\right\rangle\right)$ & $P_{1}(G F(2) \times G F(2))$ \\
\hline TQ & $\begin{array}{l}\text { набор из пяти взаимно } \\
\text { некоммутирующих } \\
\text { операторов }\end{array}$ & $\begin{array}{l}\text { набор из шести операторов, } \\
\text { коммутирующих с данным }\end{array}$ & $\begin{array}{l}\text { девять операторов } \\
\text { квадрата Мермина }\end{array}$ \\
\hline
\end{tabular}

внутреннюю природу этих явлений, но также открывает совершенно новые перспективы их приложений и далеко простирающиеся возможности алгебро-геометрического моделирования аналогов этих систем в старших размерностях [24], [25].

Благодарности. М. Санига признателен профессору Г. Хавличеку (Vienna University of Technology), привлекшему наше внимание к описанию обобщенного квадрата порядка два в виде проективной прямой над жордановой системой указанного кольца. Работа была частично поддержана Агенством по поддержке науки и технологий (Республика Словакия, контракт № APVT-51-012704), проектом VEGA (Республика Словакия, контракт № 2/6070/26), международным проектом ECO-NET (Франция, грант № 12651NJ "Геометрии над конечными кольцами и свойства взаимно несвязанных базисов") и Академией наук Республики Чехия (проект 1ET400400410).

\section{Список литературы}

[1] A. Blunck, H. Havlicek, Abh. Math. Sem. Univ. Hamburg, 70 (2000), 287-299.

[2] A. Blunck, H. Havlicek, Math. Pannon., 14:1 (2003), 113-127.

[3] A. Blunck, H. Havlicek, Aequationes Math., 69 (2005), 146-163.

[4] H. Havlicek, Quad. Sem. Matem. di Brescia, 11 (2006), 1-63; http://www.geometrie.tuwien.ac.at/havlicek/pdf/dd-laguerre.pdf.

[5] A. Herzer, "Chain geometries", Handbook of Incidence Geometry, ed. F. Buekenhout, Elsevier, Amsterdam, 1995, 781-842.

[6] M. Saniga, M. Planat, M. R. Kibler, P. Pracna, Chaos Solitons Fractals, 33 (2007), 1095-1102; arXiv: math.AG/0605301.

[7] M. Saniga, M. Planat, Chaos Soliton Fractals, 37 (2008), 337-345; arXiv: math. AG/0604307.

[8] М. Санига, М. Плана, ТМФ, 151:1 (2007), 44-53; arXiv: quant-ph/0603051.

[9] М. Санига, М. Плана, М. Минарович, ТМФ, 151:2 (2007), 219-227; arXiv: quant-ph/0603206.

[10] M. Planat, M. Saniga, M. R. Kibler, SIGMA, 2 (2006), 066; arXiv: quant-ph/0605239.

[11] M. Saniga, M. Planat, P. Pracna, A classification of the projective lines over small rings II. Non-commutative case, arXiv: math.AG/0606500.

[12] J. B. Fraleigh, A First Course in Abstract Algebra, Addison-Wesley, Reading, MA, 1967. 
[13] B. R. McDonald, Finite Rings with Identity, Dekker, New York, 1974.

[14] R. Raghavendran, Compos. Math., 21 (1969), 195-229.

[15] C. Nöbauer, The book of the rings, http://www.algebra.uni-linz.ac.at/ noebsi/ pub/rings.ps, 2000, part I.

[16] H. Van Maldeghem, Generalized Polygons, Birkhäuser, Basel, 1998.

[17] B. Polster, H. Van Maldeghem, J. Combin. Theory Ser. A, 96:1 (2001), 162-179.

[18] B. Polster, A Geometrical Picture Book, Springer, New York, 1998.

[19] B. Polster, Bull. Belg. Math. Soc. Simon Stevin, 5 (1998), 417-425.

[20] B. Polster, A. E. Schroth, H. Van Maldeghem, Math. Intelligencer, 23 (2001), 33-47.

[21] J. Lawrence, Č. Brukner, A. Zeilinger, Phys. Rev. A, 65 (2002), 032320.

[22] S.E. Payne, J.A. Thas, Finite Generalized Quadrangles, Pitman, Boston, London, Melbourne, 1984.

[23] H. Havlicek, Mitt. Math. Ges. Hamburg, 26 (2007), 75-94; http://www.geometrie.tuwien.ac.at/havlicek/pub/pentazyklisch.pdf.

[24] M. Saniga, M. Planat, Adv. Studies Theor. Phys., 1:1-4 (2007), 1-4; arXiv: quant-ph/0612179.

[25] M. Planat, M. Saniga, Quantum Inf. Comput., 8:1-2 (2008), 127-146; arXiv: quant-ph/0701211.

Поступила в редакцию 12.12.2006, после доработки 20.03.2007 